\title{
Designing a Fuzzy Expert System to Evaluate Alternatives in Fuzzy Analytic Hierarchy Process
}

\author{
Hamed Fazlollahtabar ${ }^{1}$, Hamid Eslami ${ }^{2}$, Hamidreza Salmani ${ }^{2}$ \\ ${ }^{1}$ Mazandaran University of Science and Technology, Babol, Iran; ${ }^{2}$ Science and Research Campus, Islamic Azad University, Member \\ of Young Researchers Club, Tehran, Iran. \\ Email: hamed@ustmb.ac.ir
}

Received June 23 ${ }^{\text {rd }}$, 2009; revised July $18^{\text {th }}$, 2009; accepted July 25 ${ }^{\text {th }}, 2009$.

\begin{abstract}
This paper concerns with proposing a fuzzy logic based expert system to breakthrough the problem of alternatives evaluation in Analytic Hierarchy Process (AHP). AHP as a multi criteria decision aid helped decision makers for analyzing and prioritizing the alternatives in a hierarchical structure. During times AHP encountered some problems. Hence, fuzzy analytic hierarchy process (FAHP) and some other extensions of AHP have been configured to solve those problems.
\end{abstract}

Keywords: Multi Criteria Decision Making (MCDM), Analytic Hierarchy Process (AHP), Expert System

\section{Introduction}

Analytic hierarchy process (AHP) [1] has been widely used as a useful multiple criteria decision making (MCDM) tool or a weight estimation technique in many areas such as selection, evaluation, planning and development, decision making, forecasting, and so on [2]. The AHP is expressed by a unidirectional hierarchical relationship amongst decision levels. The top element of the hierarchy is the overall goal for the decision model. The hierarchy decomposes to a more specific criterion on a level and each criterion may be related to some subcriteria. The AHP separates complex decision problems into elements within a simplified hierarchical system.

The AHP usually consists of three stages of problem solving: decomposition, comparative judgments, and synthesis of priority. The decomposition stage aims at the construction of a hierarchical network to represent a decision problem, with the top level representing the overall objectives and the lower levels representing the criteria, subcriteria, and alternatives. With comparative judgments, users are requested to set up a comparison matrix at each hierarchy by comparing pairs of criteria or subcriteria. A scale of values ranging from 1 (Equally Preferred) to 9 (Extremely Preferred), is used to express the users preferences. Finally, in the synthesis of priority stage, each comparison matrix is then solved by an eigenvector method for determining the importance of the criteria and alternative performance.

One major advantage of AHP is its applicability to the problems of group decision-making. In a group decision setting, each participant is required to set up the preference of each alternative by the AHP and the collective views of the participants are used to obtain an average weighting of each alternative.

The traditional AHP requires crisp judgments. However, due to the complexity and uncertainty involved in real world decision problems, a decision maker (DM) may sometimes feel more confident to provide fuzzy judgments than crisp comparisons. A number of methods have been developed to handle fuzzy comparison matrices. For example, Van Laarhoven and Pedrycz [3] suggested a fuzzy logarithmic least squares method (LLSM) to obtain triangular fuzzy weights from a triangular fuzzy comparison matrix. Wang et al. [4] presented a modified fuzzy LLSM.

Buckley [5] utilized the geometric mean method to calculate fuzzy weights. Chang [6] proposed an extent analysis method, which derives crisp weights for fuzzy comparison matrices. Xu [7] brought forward a fuzzy least squares priority method (LSM). Mikhailov [8] developed a fuzzy preference programming method (PPM), which also derives crisp weights from fuzzy comparison matrices. Csutora and Buckley [9] came up with a Lambda-Max method, which is the direct fuzzification of the well-known kmax method.

Among the above approaches, the extent analysis method has been employed in quite a number of applications [10-28] due to its computational simplicity. However, such a method is found unable to derive the true 
weights from a fuzzy or crisp comparison matrix. The weights determined by the extent analysis method do not represent the relative importance of decision criteria or alternatives at all. Therefore, it should not be used as a method for estimating priorities from a fuzzy pairwise comparison matrix. The purpose of this paper is to show by examples that the priority vectors determined by the extent analysis method do not represent the relative importance of decision criteria or alternatives and that the misapplication of the extent analysis method to fuzzy AHP problems may lead to a wrong decision to be made and some useful decision information such as decision criteria and fuzzy comparison matrices not to be considered. We illustrate these problems to avoid any possible misapplications in the future. Here, we compare the Fuzzy AHP with a proposed expert system and illustrate our proposed expert system in an example.

\section{Review of the Extent Analysis Method on Fuzzy AHP}

A triangular fuzzy number is represented by $\tilde{a}=$ $(l, m, u)$, with the membership function, $\mu_{\tilde{a}}(x)$, defined by the expression,

$$
\mu_{\tilde{a}}(x)=\left\{\begin{array}{ll}
1-\left(\frac{m-x}{l}\right) & x \leq m \\
1-\left(\frac{x-m}{u}\right) & x>m
\end{array}\right\}
$$

where $m$ is the center, $l$ is the left spread and $u$ is the right spread. For two triangular fuzzy number $\tilde{M}_{1}=$ $\left(l_{1}, m_{1}, u_{1}\right)$ and $\tilde{M}_{2}=\left(l_{2}, m_{2}, u_{2}\right)$ the fuzzy operations are defined as follows:

$$
\begin{gathered}
\tilde{M}_{1}+\tilde{M}_{2}=\left(l_{1}+l_{2}, m_{1}+m_{2}, u_{1}+u_{2}\right) \\
\tilde{M}_{1} \times \tilde{M}_{2}=\left(l_{1} \times l_{2}, m_{1} \times m_{2}, u_{1} \times u_{2}\right) \\
\tilde{M}_{1}^{-1}=\left(\frac{1}{u_{1}}, \frac{1}{m_{1}}, \frac{1}{l_{1}}\right), \quad \tilde{M}_{2}^{-1}=\left(\frac{1}{u_{2}}, \frac{1}{m_{2}}, \frac{1}{l_{2}}\right)
\end{gathered}
$$

Consider a triangular fuzzy comparison matrix expressed by

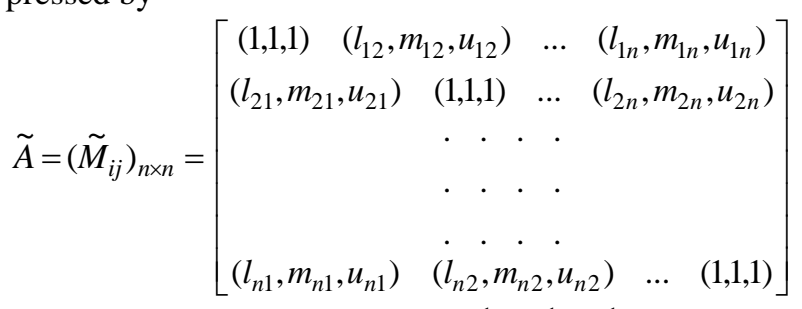

where $\tilde{a}_{i j}=\left(l_{i j}, m_{i j}, u_{i j}\right)=\tilde{a}_{j i}^{-1}=\left(\frac{1}{u_{j i}}, \frac{1}{m_{j i}}, \frac{1}{l_{j i}}\right)$, for $i, j=$ $1, \ldots, n$ and $i \neq j$.

To calculate a priority vector of the above triangular fuzzy comparison matrix, Chang [9] suggested an extent analysis method, which is summarized as follows.
Firstly, sum up each row of the fuzzy comparison matrix $\tilde{A}$ by fuzzy arithmetic operations:

$$
R S_{i}=\sum_{j=1}^{n} \tilde{M}_{i j}=\left(\sum_{j=1}^{n} \tilde{l}_{i j}, \sum_{j=1}^{n} \tilde{m}_{i j}, \sum_{j=1}^{n} \tilde{u}_{i j},\right), i=1, \ldots, n .
$$

Secondly, normalize the above row sums by

$$
\tilde{S}_{i}=\frac{R S_{i}}{\sum_{j=1}^{n} R S_{j}}=\left(\frac{\sum_{j=1}^{n} \tilde{l}_{i j}}{\sum_{k=1}^{n} \sum_{j=1}^{n} u_{k j}}, \frac{\sum_{j=1}^{n} \tilde{m}_{i j}}{\sum_{k=1}^{n} \sum_{j=1}^{n} m_{k j}}, \frac{\sum_{j=1}^{n} \tilde{u}_{i j}}{\sum_{k=1}^{n} \sum_{j=1}^{n} l_{k j}}\right), \quad i=1, \ldots, n .
$$

Thirdly, compute the degree of possibility of $\tilde{S}_{i} \geq \tilde{S}_{j}$ by the following equation:

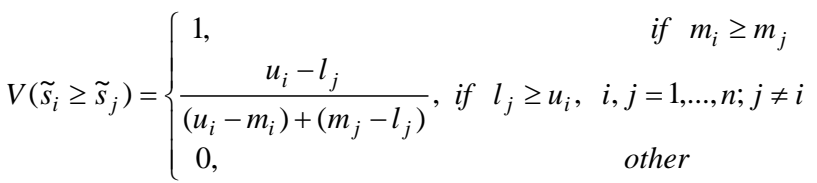
where $\tilde{S}_{i}=\left(l_{i}, m_{i}, u_{i}\right)$ and $\tilde{S}_{j}=\left(l_{j}, m_{j}, u_{j}\right)$. The definition of possibility degree is shown in Figure 1.

Fourthly, calculate the degree of possibility of $\tilde{S}_{i}$ over all the other $(n-1)$ fuzzy numbers by $V\left(\tilde{S}_{i} \geq \tilde{S}_{j} \mid j=1\right.$, $\left.\ldots, n ; j \neq i)=\min _{j \in\{1, \ldots, n, j, j \neq i} V\left(\tilde{S}_{i} \geq \tilde{S}_{j}\right), \quad i=1, \ldots, n\right)$.

Finally, define the priority vector $W=\left(w_{1}, \ldots, w_{n}\right)^{T}$ of the fuzzy comparison matrix $\tilde{A}$ as

$$
w_{i}=\frac{V\left(\tilde{S}_{i} \geq \tilde{S}_{j} \mid j=1, \ldots, n ; j \neq i\right)}{\sum_{k=1}^{n} V\left(\tilde{S}_{k} \geq \tilde{S}_{j} \mid j=1, \ldots, n ; j \neq k\right)}, \quad i=1, \ldots, n .
$$

It must be pointed out that the normalization formula is wrong. The correct normalization formula for a set of triangular fuzzy weights should be as follows:

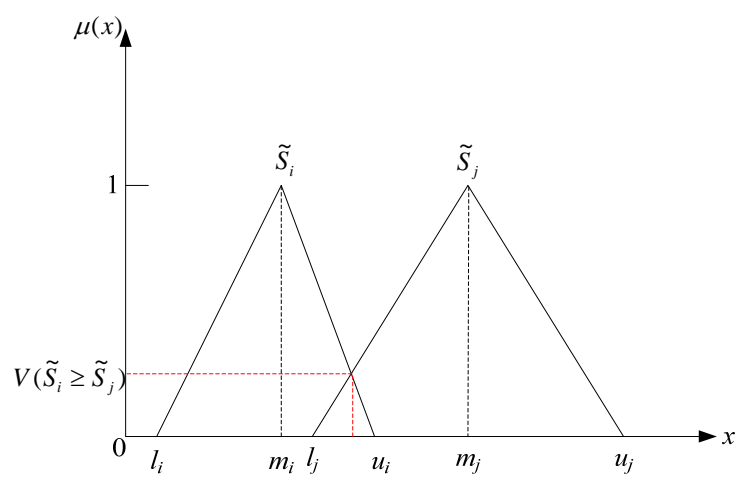

Figure 1. Definition of the degree of possibility of $V\left(\tilde{S}_{i} \geq \tilde{S}_{j}\right)$ 


$$
\begin{gathered}
\tilde{S}_{i}=\frac{R S_{i}}{\sum_{j=1}^{n} R S_{j}}=\left(\frac{\sum_{j=1}^{n} l_{i j}}{\sum_{j=1}^{n} \tilde{l}_{i j}+\sum_{k=1, k \neq i}^{n} \sum_{j=1}^{n} u_{k j}}, \frac{\sum_{j=1}^{n} m_{i j}}{\sum_{k=1}^{n} \sum_{j=1}^{n} m_{k j}},\right. \\
\left.\frac{\sum_{j=1}^{n} u_{i j}}{\sum_{j=1}^{n} u_{i j}+\sum_{k=1, k \neq i}^{n} \sum_{j=1}^{n} l_{k j}}\right), i=1, \ldots, n .
\end{gathered}
$$

Although Fuzzy AHP solved some of the problems of AHP, but still some problems arises:

Problem 1. The extent analysis method may assign a zero weight to a decision criterion or alternative, leading to the criterion or alternative not to be considered in decision analysis.

Problem 2. The weights determined by the extent analysis method do not represent the relative importance of decision criteria or alternatives and cannot be used as their priorities.

Problem 3. The extent analysis method may make a wrong decision and select the worst decision alternative as the best one when it is misused for solving a fuzzy AHP problem.

Problem 4. The extent analysis method cannot make full use of all the fuzzy comparison matrices information and may cause some useful fuzzy comparison matrices information to be wasted when it assigns an irrational zero weight to some useful decision criteria or sub-criteria.

Therefore, we propose an expert system which functions based on fuzzy logic, to improve decision making in uncertainties.

\section{Fuzzy Logic}

Fuzzy Logic (FL) is a problem-solving control system methodology that lends itself to implementation in systems ranging from simple, small, embedded microcontrollers to large, networked, multi-channel PC or workstation-based data acquisition and control systems. It can be implemented in hardware, software, or a combination of both. FL provides a simple way to arrive at a definite conclusion based upon vague, ambiguous, imprecise, noisy, or missing input information. FL's approach to control problems mimics how a person would make decisions, only much faster.

FL incorporates a simple, rule-based IF X AND Y THEN Z approach to a solving control problem rather than attempting to model a system mathematically. The FL model is empirically-based, relying on an operator's experience rather than their technical understanding of the system.

FL requires some numerical parameters in order to operate such as what is considered significant error and significant rate-of-change-of-error, but exact values of these numbers are usually not critical unless very responsive performance is required in which case empirical tuning would determine them. For example, a simple temperature control system could use a single temperature feedback sensor whose data is subtracted from the command signal to compute "error" and then timedifferentiated to yield the error slope or rate-of-changeof-error, hereafter called "error-dot". Error might have units of degs $\mathrm{F}$ and a small error considered to be $2 \mathrm{~F}$ while a large error is $5 \mathrm{~F}$. The "error-dot" might then have units of degs/min with a small error-dot being $5 \mathrm{~F} / \mathrm{min}$ and a large one being $15 \mathrm{~F} / \mathrm{min}$. These values don't have to be symmetrical and can be "tweaked" once the system is operating in order to optimize performance. Generally, FL is so forgiving that the system will probably work the first time without any tweaking.

FL works as follows:

1) Define the control objectives and criteria: What am I trying to control? What do I have to do to control the system? What kind of response do I need? What are the possible (probable) system failure modes?

2) Determine the input and output relationships and choose a minimum number of variables for input to the FL engine (typically error and rate-of-change-of-error).

3) Using the rule-based structure of FL, break the control problem down into a series of IF X AND/OR Y THEN Z rules that define the desired system output response for given system input conditions. The number and complexity of rules depends on the number of input parameters that are to be processed and the number fuzzy variables associated with each parameter. If possible, use at least one variable and its time derivative. Although it is possible to use a single, instantaneous error parameter without knowing its rate of change, this cripples the system's ability to minimize overshoot for a step inputs.

4) Create FL membership functions that define the meaning (values) of Input/Output terms used in the rules.

5) Create the necessary pre- and post-processing FL routines if implementing in $\mathrm{S} / \mathrm{W}$, otherwise program the rules into the $\mathrm{FL} \mathrm{H/W}$ engine.

6) Test the system, evaluate the results, tune the rules and membership functions, and retest until satisfactory results are obtained.

In 1973, Professor Lotfi Zadeh proposed the concept of linguistic or "fuzzy" variables. Think of them as linguistic objects or words, rather than numbers. The sensor input is a noun, e.g. "temperature”, "displacement”, "velocity", "flow", "pressure", etc. Since error is just the difference, it can be thought of the same way. The fuzzy variables themselves are adjectives that modify the variable (e.g. "large positive" error, "small positive" error, "zero" error, "small negative" error, and "large negative" error). As a minimum, one could simply have "positive", "zero", and "negative" variables for each of the parame- 
ters. Additional ranges such as "very large" and "very small" could also be added to extend the responsiveness to exceptional or very nonlinear conditions, but aren't necessary in a basic system. Here, using fuzzy logic we define some rules that help the student to select the optimal department, course and teacher based on his age, average grade and skills.

\section{Expert Systems}

Knowledge-based systems are systems based on the methods and techniques of Artificial Intelligence. Their core components are the knowledge base and the inference mechanisms. Some particular types of knowledge-based systems are expert systems, case-based reasoning systems and neural networks.

Expert Systems (ES) are computer programs that are derived from a branch of computer science research called Artificial Intelligence (AI). AI's scientific goal is to understand intelligence by building computer programs that exhibit intelligent behavior. It is concerned with the concepts and methods of symbolic inference, or reasoning, by a computer, and how the knowledge used to make those inferences will be represented inside the machine.

Of course, the term intelligence covers many cognitive skills, including the ability to solve problems, learn, and understand language; AI addresses all of those. But most progress to date in AI has been made in the area of problem solving; concepts and methods for building programs that reason about problems rather than calculate a solution.

AI programs that achieve expert-level competence in solving problems in task areas by bringing to bear a body of knowledge about specific tasks are called knowledge-based or expert systems. Often, the term expert systems is reserved for programs whose knowledge base contains the knowledge used by human experts, in contrast to knowledge gathered from textbooks or non-experts. More often, the two terms, expert systems (ES) and knowledge-based systems (KBS), are used synonymously. Taken together, they represent the most widespread type of AI application. The area of human intellectual endeavor to be captured in an expert system is called the task domain. Task refers to some goal-oriented, problem-solving activity. Domain refers to the area within which the task is being performed. Typical tasks are diagnosis, planning, scheduling, configuration and design.

Building an expert system is known as knowledge engineering and its practitioners are called knowledge engineers. The knowledge engineer must make sure that the computer has all the knowledge needed to solve a problem. The knowledge engineer must choose one or more forms in which to represent the required knowledge as symbol patterns in the memory of the computer, that is, he (or she) must choose a knowledge representation. He must also ensure that the computer can use the knowledge efficiently by selecting from a handful of reasoning methods.

Every expert system consists of two principal parts: the knowledge base; and the reasoning, or inference, engine. The knowledge base of expert systems contains both factual and heuristic knowledge. Factual knowledge is that knowledge of the task domain that is widely shared, typically found in textbooks or journals, and commonly agreed upon by those knowledgeable in the particular field.

Today there are two ways to build an expert system. They can be built from scratch, or built using a piece of development software known as a "tool" or a "shell". Before we discuss these tools, let's briefly discuss what knowledge engineers do. Though different styles and methods of knowledge engineering exist, the basic approach is the same: a knowledge engineer interviews and observes a human expert or a group of experts and learns what the experts know, and how they reason with their knowledge. The engineer then translates the knowledge into a computer-usable language, and designs an inference engine, a reasoning structure, that uses the knowledge appropriately. He also determines how to integrate the use of uncertain knowledge in the reasoning process, and what kinds of explanation would be useful to the end user.

Next, the inference engine and facilities for representing knowledge and for explaining are programmed, and the domain knowledge is entered into the program piece by piece. It may be that the inference engine is not just right; the form of knowledge representation is awkward for the kind of knowledge needed for the task; and the expert might decide the pieces of knowledge are wrong. All these are discovered and modified as the expert system gradually gains competence.

The discovery and accumulation of techniques of machine reasoning and knowledge representation is generally the work of artificial intelligence research. The discovery and accumulation of knowledge of a task domain is the province of domain experts. Domain knowledge consists of both formal, textbook knowledge, and experiential knowledge - the expertise of the experts.

Compared to the wide variation in domain knowledge, only a small number of AI methods are known that are useful in expert systems. That is, currently there are only a handful of ways in which to represent knowledge, or to make inferences, or to generate explanations. Thus, systems can be built that contain these useful methods without any domain-specific knowledge. Such systems are known as skeletal systems, shells, or simply AI tools.

Building expert systems by using shells offers significant advantages. A system can be built to perform a unique task by entering into a shell all the necessary knowledge about a task domain. The inference engine 
that applies the knowledge to the task at hand is built into the shell. If the program is not very complicated and if an expert has had some training in the use of a shell, the expert can enter the knowledge himself.

Many commercial shells are available today, ranging in size from shells on PCs, to shells on workstations, to shells on large mainframe computers. They range in price from hundreds to tens of thousands of dollars, and range in complexity from simple, forward-chained, rule-based systems requiring two days of training to those so complex that only highly trained knowledge engineers can use them to advantage. They range from general-purpose shells to shells custom-tailored to a class of tasks, such as financial planning or real-time process control.

Although shells simplify programming, in general they don't help with knowledge acquisition. Knowledge acquisition refers to the task of endowing expert systems with knowledge, a task currently performed by knowledge engineers. The choice of reasoning method, or a shell, is important, but it isn't as important as the accumulation of high-quality knowledge. The power of an expert system lies in its store of knowledge about the task domain - the more knowledge a system is given, the more competent it becomes. Primarily, the benefits of ESs to end users include:

- A speed-up of human professional or semi-professional work - typically by a factor of ten and sometimes by a factor of a hundred or more.

- Within companies, major internal cost savings. For small systems, savings are sometimes in the tens or hundreds of thousands of dollars; but for large systems, often in the tens of millions of dollars and as high as hundreds of millions of dollars. These cost savings are a result of quality improvement, a major motivation for employing expert system technology.

- Improved quality of decision making. In some cases, the quality or correctness of decisions evaluated after the fact show a ten-fold improvement.

- Preservation of scarce expertise. ESs are used to preserve scarce know-how in organizations, to capture the expertise of individuals who are retiring, and to preserve corporate know-how so that it can be widely distributed to other factories, offices or plants of the company.

- Introduction of new products. A good example of a new product is a pathology advisor sold to clinical pathologists in hospitals to assist in the diagnosis of diseased tissue.

An expert system tool, or shell, is a software development environment containing the basic components of expert systems. Associated with a shell is a prescribed method for building applications by configuring and instantiating these components. Some of the generic components of a shell are shown in Figure 2 and described below. The core components of expert systems are the knowledge base and the reasoning engine.

Knowledge base: A store of factual and heuristic knowledge. An ES tool provides one or more knowledge representation schemes for expressing knowledge about the application domain. Some tools use both frames (objects) and IF-THEN rules. In PROLOG the knowledge is represented as logical statements.

Reasoning engine: Inference mechanisms for manipulating the symbolic information and knowledge in the knowledge base to form a line of reasoning for solving a problem. The inference mechanism can range from simple modus pones backward chaining of IF-THEN rules to case-based reasoning.

Knowledge acquisition subsystem: A subsystem to help experts build knowledge bases. Collecting knowledge needed to solve problems and build the knowledge base continues to be the biggest bottleneck in building expert systems.

Explanation subsystem: A subsystem that explains the system's actions. The explanation can range from how the final or intermediate solutions were arrived at to justifying the need for additional data.

User interface: The means of communication with the user. The user interface is generally not a part of the ES technology, and was not given much attention in the past. However, it is now widely accepted that the user interface can make a critical difference in the perceived utility of a system regardless of the system's performance.

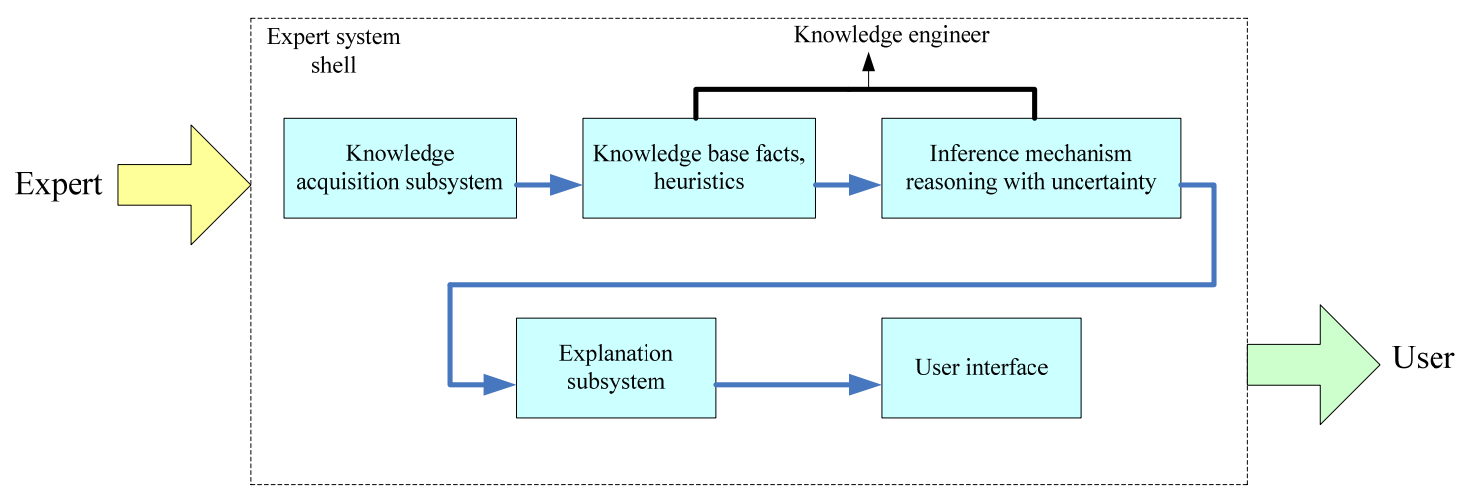

Figure 2. Basic components of expert system tools 


\section{Comparison between Expert System and Fuzzy AHP}

Expert system has been applied for ranking [29]. Expert system in comparison with fuzzy AHP has the following advantages:

- The alternatives are analyzed using quantitative and qualitative criteria without normalization process

- More than seven alternatives can be processed against AHP which encountered problems in pairwise comparisons [30].

- By entrance of new alternatives, the ranking of the alternatives do not change

- The fuzzy expert system is able to consider a standard in evaluating the alternatives

- It is possible to apply group decision making of the experts in evaluating the alternatives

- The capability of sensitivity analysis for all the alternatives

- No limitation for evaluating many criteria

- The mistakes in computations such as the zero result will not occur in expert system

- The possibility of evaluating the alternatives using both quantitative and qualitative criteria

- The possibility of evaluating the alternatives while some information about some criteria are missing

- The possibility of keeping the same membership during the process of decision making [31].

In an expert system a membership function is proposed for criteria regarding to the experts idea. To propose an expert system the following steps should be taken:

1) Determining the objective, alternatives and criteria

2) Identifying the input and output variables

3) Proposing membership functions for input and output variables

4) Proposing rules to determine the relations between inputs and outputs

5) Selecting an appropriate inference mechanism

6) Placement of alternatives corresponding to each criteria

7) Extracting the evaluation result by the proposed expert system

8) Sensitivity analysis of evaluated alternatives

Net section presents a numerical illustration to indicate the application of the proposed expert system.

\section{Numerical Illustrations}

Here, we illustrate the proposed expert system in prioritizing four brands of mobile phone. We analyze HAD, IC, TA, HAM as alternatives using the criteria services, power of antenna, prestige, and price. The hierarchy of the model is shown in Figure 3.

The linguistic variables for criteria and their corresponded membership functions are as follows (Figures 4-9).

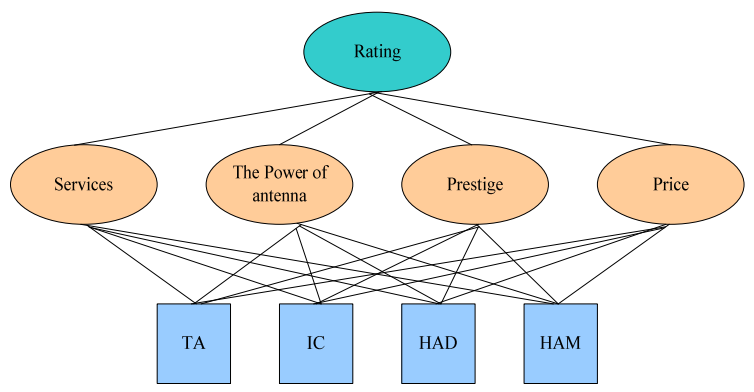

Figure 3. The hierarchy of the model

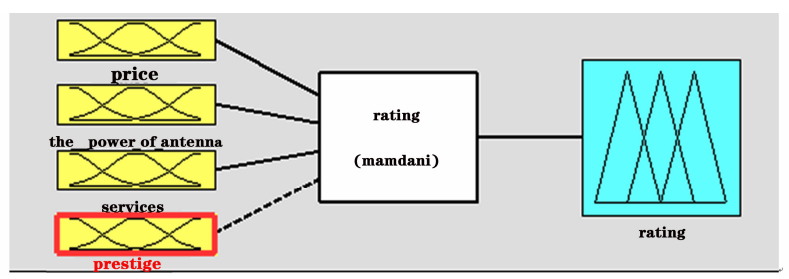

Figure 4. The inputs and outputs

Considering the experts the price has a Gaussian membership function with minimum price of 5000 and maximum of 700000 .

For the power of antenna linguistic triangular fuzzy number (high, medium, low) is considered.

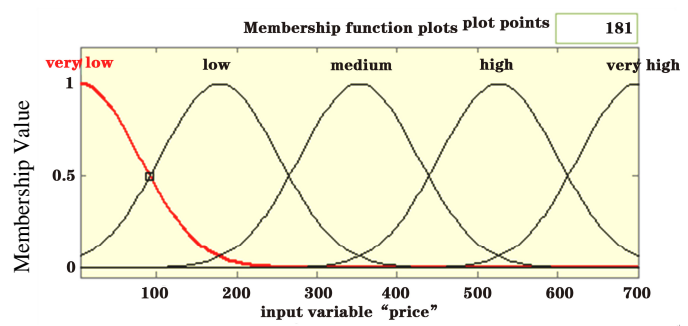

Figure 5. Price membership function

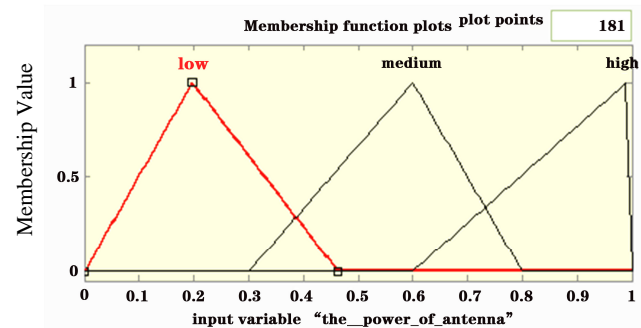

Figure 6. The power of antenna membership function

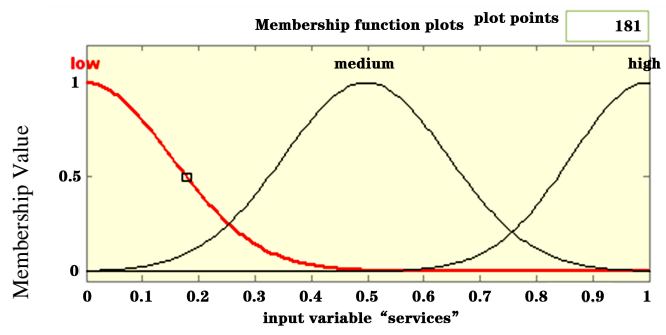

Figure 7. Services membership function 


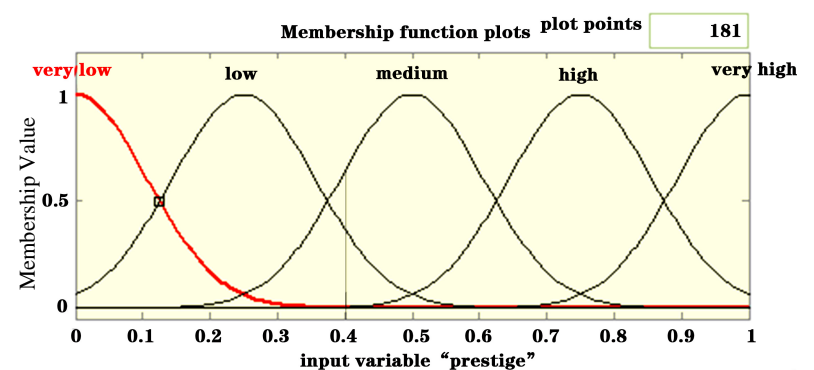

Figure 8. Prestige membership function

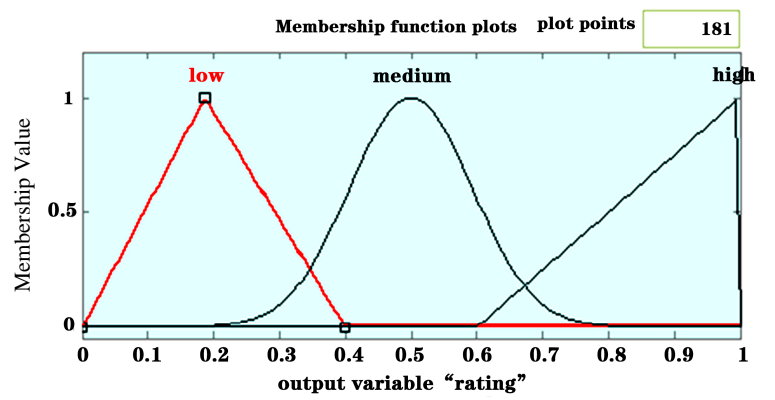

Figure 9. Rating membership function

The output of the system which is the evaluation result, is a combined linguistic fuzzy number with a Gaussian membership function for medium and triangular fuzzy membership function for high and low.

Regarding to the experts and taking the criteria into considerations, the following rules are derived:

1-If price is high Then rating medium.

2-If price is very low Then rating low.

3-If services is high Then rating high.

4-If services is medium Then rating low.
5-If services is low Then rating low.

6-If services is high Then rating high.

7-If the_power_of_antenna is high Then rating

high.

8-If the_power_of_antenna is low Then rating

low.

9-If the_power_of_antenna is medium Then rating low.

10-If price is medium Then rating high.

11-If price is very high Then rating low.

12-If price is low Then rating high.

13-If prestige is very low Then rating low.

14-If prestige is medium Then rating me-

dium.

15-If prestige is very high Then rating high

Regarding to the proposed rules of the expert system, we evaluate the alternatives as follows. To facilitate the computations MATLAB package has been applied (Appendix A).

TA

Price: 200

Services: medium

Power of antenna: high

Prestige: medium

Output: 0.518 IC

The graphical presentation is shown in Figure 10.

Price: 15

Services: low

Power of antenna: high

Prestige: low

Output: 0.51

The graphical presentation is shown in Figure 11.
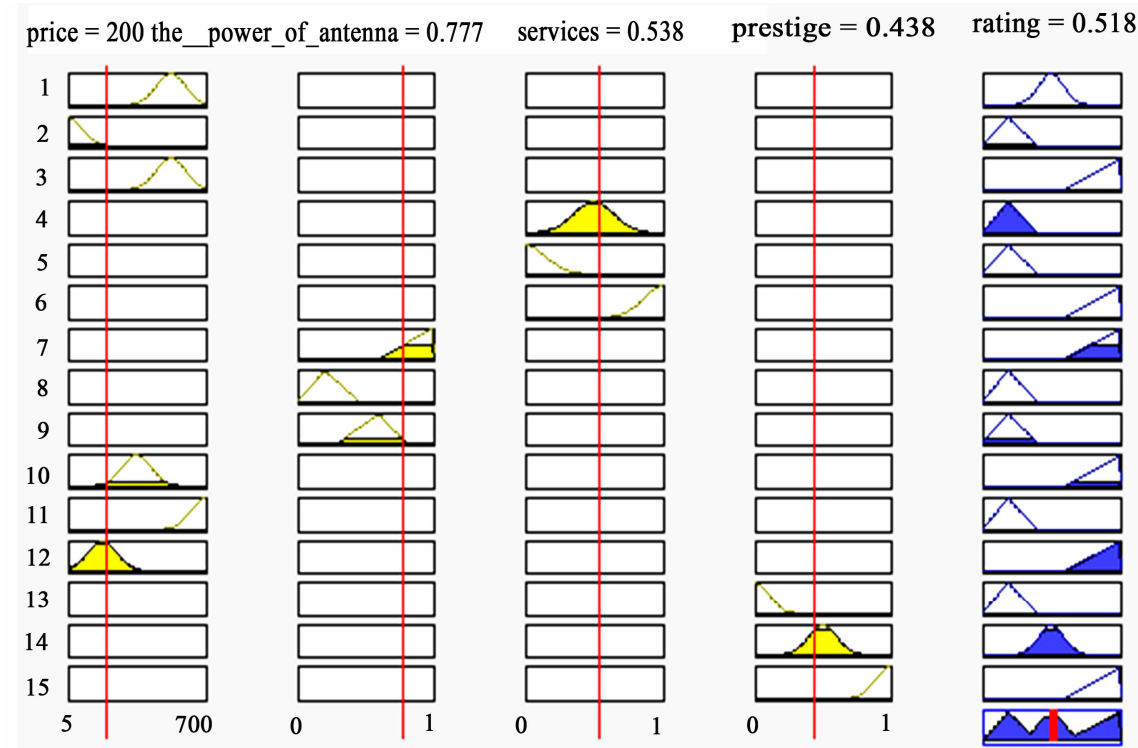

Figure 10. The rule viewer for TA 

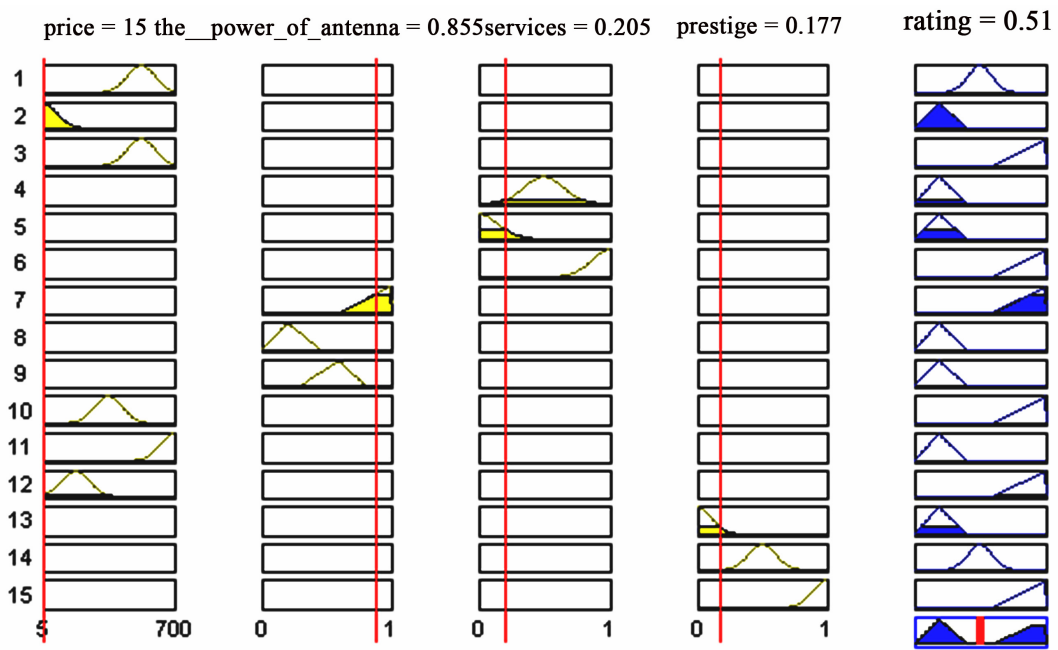

Figure 11. The rule viewer for IC
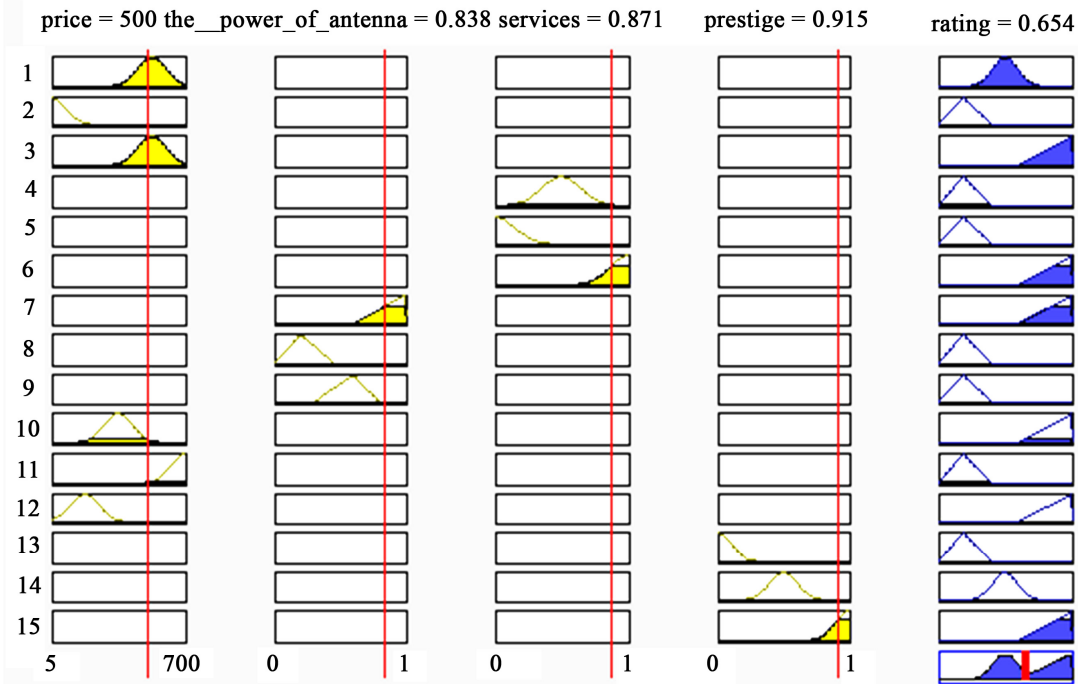

Figure 12. The rule viewer for HAD

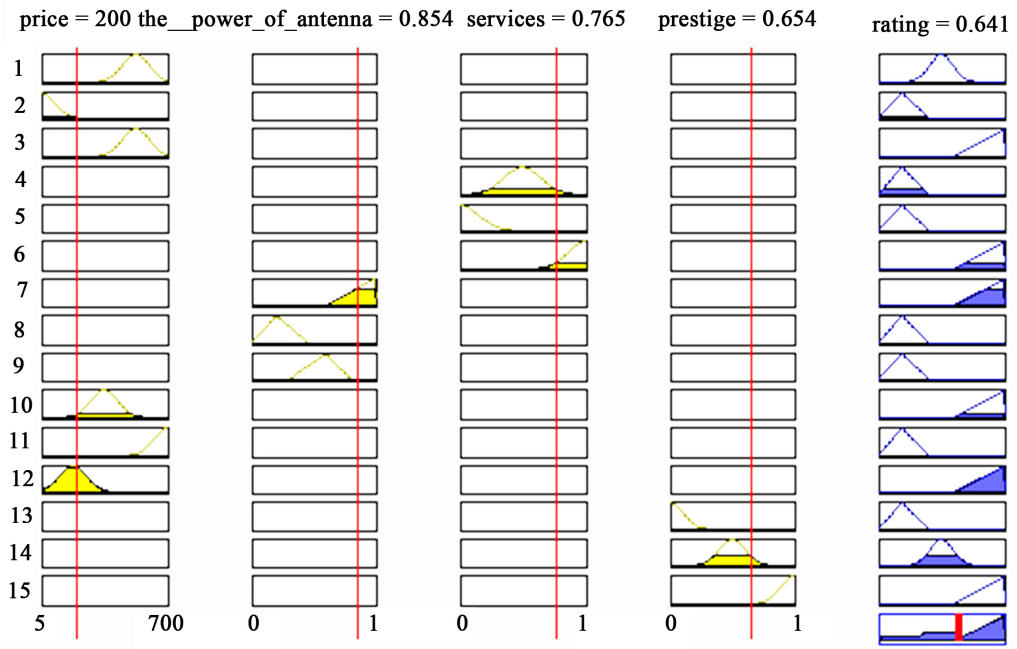

Figure 13. The rule viewer for HAM 
HAD

Price: 500

Services: high

Power of antenna: high

Prestige: very high

Output: 0.654

The graphical presentation is shown in Figure 12. HAM

Price: 200

Services: high

Power of antenna: high

Prestige: high

Output: 0.641

The graphical presentation is shown in Figure 13.

The ranking indicates the importance degree of each mobile brand.

\section{Conclusions}

In this paper, we developed a fuzzy expert system on the basis of rule base fuzzy logic to overcome the problems in AHP and Fuzzy AHP. The advantages of using expert system to prioritize the alternatives in comparison with fuzzy AHP are discussed. To present the validity and effectiveness of the proposed expert system a numerical example is illustrated.

\section{REFERENCES}

[1] T. L. Saaty, "Multicriteria Decision Making: The Analytic Hierarchy Process,” RWS Publications, Pittsburgh, 1988.

[2] O. S. Vaidya and S. Kumar, "Analytic Hierarchy Process: An Overview of Applications," European Journal of Operational Research, Vol. 169, 2006, pp. 1-29.

[3] P. J. M. Van Laarhoven and W. Pedrycz, "A Fuzzy Extension of Saaty's Priority Theory,” Fuzzy Sets and Systems, Vol. 11, 1983, pp. 229-241.

[4] Y. M. Wang, T. M. S. Elhag and Z. S. Hua, “A Modified Fuzzy Logarithmic Least Squares Method for Fuzzy Analytic Hierarchy Process,” Fuzzy Sets and Systems, Vol. 157, 2006, pp. 3055-3071.

[5] J. J. Buckley, "Fuzzy Hierarchical Analysis,” Fuzzy Sets and Systems, Vol. 17, 1985, pp. 233-247.

[6] D. Y. Chang, "Applications of the Extent Analysis Method on Fuzzy AHP," European Journal of Operational Research, Vol. 95, 1996, pp. 649-655.

[7] R. Xu, "Fuzzy Least-Squares Priority Method in the Analytic Hierarchy Process,” Fuzzy Sets and Systems, Vol. 112, 2000, pp. 359-404.

[8] L. Mikhailov, "Deriving Priorities from Fuzzy Pairwise Comparison Judgments,” Fuzzy Sets and Systems, Vol. 134, 2003, pp. 365-385.

[9] R. Csutora, and J. J. Buckley, "Fuzzy Hierarchical Analysis: The Lamda-Max Method,” Fuzzy Sets and Systems, Vol. 120, 2001, pp. 181-195.
[10] F. T. Bozbura and A. Beskese, "Prioritization of Organizational Capital Measurement Indicators Using Fuzzy AHP,” International Journal of Approximate Reasoning, Vol. 44, 2007, pp. 124-147.

[11] F. T. Bozbura, A.Beskese and C. Kahraman, "Prioritization of Human Capital Measurement Indicators Using Fuzzy AHP,” Expert Systems with Applications, Vol. 32, 2007, pp. 1100-1112.

[12] C. E. Bozdag, C. Kahraman and D. Ruan, "Fuzzy Group Decision Making for Selection among Computer Integrated Manufacturing Systems," Computers in Industry, Vol. 51, 2003, pp. 13-29.

[13] G. Bu“yu"ko"zkan, "Multi-Criteria Decision Making for E-Marketplace Selection,” Internet Research, Vol. 14, No. 2, 2004, pp. 139-154.

[14] G. Büyu“ko“zkan, T. Ertay, C. Kahraman and D. Ruan, "Determining the Importance Weights for the Design Requirements in the House of Quality Using the Fuzzy Analytic Network Approach," International Journal of Intelligent Systems, Vol. 19, 2004, pp. 443-461.

[15] G. Bu“yu“ko“zkan, C. Kahraman and D. Ruan, “A Fuzzy Multi-Criteria Decision Approach for Software Development Strategy Selection," International Journal of General Systems, Vol. 33, 2004, pp. 259-280.

[16] F. T. S. Chan and N. Kumar, "Global Supplier Development Considering Risk Factors Using Fuzzy Extended AHP Based Approach,” Omega, Vol. 35, 2007, pp. 417-431.

[17] T. Ertay, G. Bu“yu“ko“zkan, C. Kahraman and D. Ruan, "Quality Function Deployment Implementation Based on Analytic Network Process with Linguistic Data: An Application in Automotive Industry,” Journal of Intelligent and Fuzzy Systems, Vol. 16, 2005, pp. 221-232.

[18] Y. C. Erensal, T. O“ ncan and M. L. Demircan, "Determining Key Capabilities in Technology Management Using Fuzzy Analytic Hierarchy Process: A Case Study of Turkey,” Information Sciences, Vol. 176, 2006, pp. 27552770.

[19] C Kahraman, U. Cebeci and D. Ruan, "Multi-Attribute Comparison of Catering Service Companies Using Fuzzy AHP: The Case of Turkey," International Journal of Production Economics, Vol. 87, 2004, pp. 171-184.

[20] C. Kahraman, U. Cebeci and Z. Ulukan, "Multi-Criteria Supplier Selection Using Fuzzy AHP,” Logistics Information Management, Vol. 16, No. 6, 2003, pp. 382-394.

[21] C. Kahraman, T. Ertay, and G. Büyu“ko“zkan, “A Fuzzy Optimization Model for QFD Planning Process Using Analytic Network Approach,” European Journal of Operational Research, Vol. 171, 2006, pp. 390-411.

[22] C. Kahraman, D. Ruan and I. Dogan, "Fuzzy Group Decision-Making for Facility Location Selection,” Information Sciences, Vol. 157, 2003, pp. 135-153.

[23] O. Kulak and C. Kahraman, "Fuzzy Multi-Attribute Selection among Transportation Companies Using Axiomatic Design and Analytic Hierarchy Process,” Information Sciences, Vol. 170, 2005, pp. 191-210.

[24] C. K. Kwong, and H. Bai, "Determining the Importance 
Weights for the Customer Requirements in QFD Using a Fuzzy AHP with an Extent Analysis Approach,” IIE Transactions, Vol. 35, 2003, pp. 619-626.

[25] Y. C. Tang and M. Beynon, “Application and Development of a Fuzzy Analytic Hierarchy Process within a Capital Investment Study," Journal of Economics and Management, Vol. 1, 2005, pp. 207-230.

[26] E. Tolga, M. L. Demircan and C. Kahraman, “Operating System Selection Using Fuzzy Replacement Analysis and Analytic Hierarchy Process," International Journal of Production Economics, Vol. 97, 2005, pp. 89-117.

[27] F. Tu“ysu“ z, and C. Kahraman, "Project Risk Evaluation Using a Fuzzy Analytic Hierarchy Process: An Application to Information Technology Projects," International Journal of Intelligent Systems, Vol. 21, No. 6, 2006, pp. 559-584.

\section{Appendix A}

Here, some useful MATLAB commands to work with the proposed fuzzy inference system (FIS) which is based on Mamdani are presented:

[System]

Name='AHP mobile'

Type='mamdani'

Version $=2.0$

NumInputs $=4$

NumOutputs $=1$

NumRules $=15$

AndMethod='min'

OrMethod='max'

ImpMethod='min'

AggMethod='max'

DefuzzMethod='centroid'
[28] K. J. Zhu, Y. Jing and D. Y. Chang, "A Discussion on Extent Analysis Method and Application of Fuzzy AHP," European Journal of Operational Research, Vol. 116, 1999, pp. 450-456.

[29] S. Malagoli, C. A. Magni and G. Mastroleo, "The Use of Fuzzy Logic and Expert Systems for Rating and Pricing Firms,” Social Science Research Network, Vol. 33, No. 11, 2007, pp. 77-120.

[30] E. Turban, J. E. Aronson and T. P. Liang, "Decision Support Systems and Intelligent Systems,” 7th Edition, Prentice Hall of India, New Delhi, 2007.

[31] Y. M. Wang, Y. Luo and Z. Hua, "On the Extent Analysis Method for Fuzzy AHP and its Applications,” European Journal of Operational Research, Vol. 186, 2008, pp. 735-747. 